\title{
Impact of Surgery on Growth, Pulmonary Functions, and Acute Pulmonary Exacerbations in Children with Non-Cystic Fibrosis Bronchiectasis
}

\author{
Nagehan Emiralioglu ${ }^{1}$ Deniz Dogru ${ }^{1}$ Songul Yalcin ${ }^{2}$ Gokcen Dılsa Tugcu ${ }^{1}$ Ebru Yalcin ${ }^{1}$ \\ Ugur Ozcelik ${ }^{1}$ Saniye Ekinci ${ }^{3}$ Nural Kiper ${ }^{1}$
}

${ }^{1}$ Hacettepe University Faculty of Medicine, Department of Pediatric Pulmonology, Ankara, Turkey

2 Hacettepe University Faculty of Medicine, Department of Social Pediatrics, Ankara, Turkey

${ }^{3}$ Hacettepe University Faculty of Medicine, Department of Pediatric Surgery, Ankara, Turkey

Address for correspondence Nagehan Emiralioglu, MD, Department of Pediatric Pulmonology, Hacettepe Universitesi Tip Fakultesi, Ihsan Dogramaci Children's Hospital, Ankara 06100, Turkey (e-mail: drnagehan@yahoo.com).

Thorac Cardiovasc Surg 2019;67:58-66.

\begin{abstract}
Keywords

- non-CF bronchiectasis

- surgery

- medical treatment

- pulmonary exacerbation

Background Treatment decisions for the management of bronchiectasis include medical treatment, such as antibiotics, chest physiotherapy, and surgical procedures. Here, we aimed to review the effect of lung resection on longitudinal growth, clinical course of patients depending on annual exacerbation rates, and pulmonary function tests (PFTs) and compare them with the results of only medically treated children with non-cystic fibrosis (non-CF) bronchiectasis.

Methods The medical records of patients with non-CF bronchiectasis were retrospectively analyzed. Patients who underwent lobectomy/segmentectomy/pneumonectomy were categorized as "surgery group" $(n=29)$. Age- and gender-matched patients who were only medically treated were selected as "medical group" $(n=33)$. Annual data of patients were included till the end of postoperative second year in the surgery group and third year of medical treatment in the medical group.

Results Mean baseline height $z$-score was lower in the surgery group, and mean baseline PFT values were all lower in the surgery group than those in the medical group $(p<0.05)$. In the surgery group, mean values of height $z$-score were $-1.68 \pm 0.92$ at the time of surgery and improved to $-1.42 \pm 1.22$ and $-1.34 \pm 1.05$ in the first and second postoperative years, respectively, and annual intravenous antibiotic requirements decreased significantly $(p<0.05)$; however, mean body mass index (BMI) $z$-score values and PFT parameters did not change significantly. In the medical group, height $z$-score mean values and PFT parameters showed nonsignificant improvement but annual exacerbation frequency, annual intravenous, and oral antibiotic requirements decreased significantly.

Conclusion Surgical management of non-CF bronchiectasis has no significant effect on BMI z-scores, annual exacerbation frequencies, oral antibiotic requirements and
\end{abstract}

received

June 1, 2017

accepted after revision

October 25, 2017

published online

December 12, 2017 (c) 2019 Georg Thieme Verlag KG

Stuttgart · New York
DOI https://doi.org/

10.1055/s-0037-1608922.

ISSN 0171-6425. 
lung function tests; but can lead to significant improvement on height z-scores and decrease need of annual intravenous antibiotic requirements for acute severe exacerbations despite small number of patients in this study.

\section{Introduction}

Bronchiectasis is a pathologic term, which is the end result of chronic inflammation and infections with resultant anatomical distortion and dilation of the airways. ${ }^{1-3}$ Bronchiectasis appears to be more common in children within developing countries and in lower socioeconomic classes. ${ }^{4}$ Recently a greater number of children are being diagnosed with bronchiectasis with the advent of high resolution computed tomography(HRCT). ${ }^{4,5}$ Non-cystic fibrosis (nonCF) bronchiectasis may be caused with a wide range of conditions, including pulmonary infections, immune deficiency, ciliary abnormalities, mechanical problems, aspiration, and idiopathic causes. ${ }^{6}$ The prognosis of non-CF bronchiectasis mostly depends on its underlying etiology. ${ }^{1}$

Treatment decisions for the management of bronchiectasis are often based on expert opinions and include pharmacologic agents, such as antibiotics, non-pharmacologic approach, such as chest physiotherapy, and surgical procedures. $^{2}$ In general, treatment goals are to control infections and improve mucociliary clearance. ${ }^{2}$ Results from adult studies are controversial and emphasize no significant effect of medical treatments on the decline of forced expiratory volume 1 second (FEV1). ${ }^{7}$ Although there is no evidence for the use of surgery in children with bronchiectasis, in symptomatic patients with prominent localized disease and not responding to appropriate therapy, resection of the most affected lobe is warranted to reduce the risk of infections. ${ }^{5,8}$ The aim of resection of destroyed tissue may prevent spreading of microorganisms from affected regions to less affected ones, thus slowing down involvement of the remaining areas. Therefore, surgery may improve quality of life in such patients.

There are no data about the long-term outcome of surgery in children with non-CF bronchiectasis, and the indications of surgery in these patients are unclear. ${ }^{1}$ In this study, our aims were: (1) to demonstrate the anthropometric data, pulmonary function tests (PFT), and clinical features of both surgically and only medically treated children with non-CF bronchiectasis when they were first diagnosed and (2) to review the effect of lung resection on longitudinal growth and clinical course of patients manifested as annual exacerbation rates and pulmonary functions and compare them with the results of only medically treated children with non-CF bronchiectasis.

\section{Methods}

\section{Subjects}

The study was conducted as a retrospective cohort study in which the medical records of patients with non-CF bronch- iectasis between December 1998 and October 2011 were reviewed.

Children with chronic cough, sputum production, and crackles in the physical examination who had bronchiectasis in HRCT of chest were included in the study. Cystic fibrosis was excluded on the basis of the clinical findings, sweat test, and/or genetic analysis. The underlying etiology of bronchiectasis was determined after performing the tests recommended in the British Thoracic Society guideline for non-CF bronchiectasis. ${ }^{9}$ Flexible bronchoscopy was performed to rule out endo-bronchial obstruction in all patients. Primary ciliary dyskinesia (PCD) diagnosis was based on the presentation of the characteristic clinical phenotype, nasal nitric oxide (NO) results, presence of ciliary ultrastructural defects (visualized by electron microscopy), and presence of abnormal ciliary function (as determined by high speed video microscopy) and/or a genetic mutation recognized to cause PCD. Immune deficiency was diagnosed based on immunoglobulin titers, nitroblue tetrazolium (NBT) blood test, and advanced immunological tests.

All patients were treated with medical treatment including airway clearance techniques, oral or intravenous antibiotics for acute pulmonary exacerbations, and bronchodilators and inhaled steroids if clinically indicated. Surgical resection of a bronchiectatic lobe(s)/segment(s) or pneumonectomy was performed in patients with bronchiectasis localized to one or more lobes who had recurrent hospitalizations due to acute pulmonary exacerbations, failure to thrive, and deterioration of PFT tests in spite of courses of regular medical treatment described above. Decision of surgical intervention was given by multidisciplinary team involving our respiratory physicians, surgeons, and radiologists depending on each patient's findings including annual hospitalization rate, growth parameters, and PFT. Ventilation-perfusion scintigraphy scan test was performed in all patients before the operation. Decision for the volume of the resected lung was based on both radiologic and scintigraphic findings as well as gross appearance of the lung on thoracotomy. Patients who had surgery continued to have regular medical treatment after the operation as well. All patients were reviewed every 3 months in our center.

Patients who underwent lobectomy/segmentectomy/ pneumonectomy and had been followed up for at least 2 years after surgery were categorized as "surgery group." Age- and gender-matched non-CF bronchiectasis patients who had been only medically treated for at least 3 years were selected as "medical group."

Data of patients in the surgery group were included in four visits totally: baseline (at time of diagnosis), at the time of surgery, in the postoperative first year, and in the postoperative second year. Data of patients in the medical group were included in four visits totally: baseline (at time 
of diagnosis) and in the first, second, and third years of medical treatment.

Age, gender, etiology of bronchiectasis, duration of symptoms, presence of clubbing, localization of bronchiectasis in chest HRCT, body weight, height, body mass index (BMI), $z$-scores of weight, height and BMI, and PFT were recorded at every visit. BMI was calculated by dividing weight in kilograms by the square of height in meters. " $z$ " scores for weight-for-age, height-for-age, and BMI-for-age were obtained from the WHO AnthroPlus packet program. ${ }^{10}$ PFT by spirometry was performed in accordance with the American Thoracic Society standards. The FEV1, FVC, and FEF2575 measurements were expressed in liters and were referred to a healthy population as a percentage of predicted value. ${ }^{11}$ Best annual PFT parameters were included for every year. Measurements after the second surgery were recorded in patients who underwent second surgery.

"Acute pulmonary exacerbation" was defined according to the British Thoracic guideline for non-CF bronchiectasis as either a change in one or more of the common symptoms of bronchiectasis (sputum volume or purulence, dyspnea, cough, and fatigue/malaise) or the onset of new symptoms (fever, pleurisy, or hemoptysis) ${ }^{9}$ and was based on the physician's diagnosis in the patients' medical records. "Annual exacerbation frequency" was defined as the number of acute pulmonary exacerbations observed in 1 year. "Annual intravenous and oral antibiotic requirement" was also recorded.

The present study was approved by a local institutional review board.

\section{Statistical Analysis}

Statistical analyses were performed using the Statistical Package for the Social Sciences (SPSS) version 23.0 (SPSS Inc., Chicago, Illinois, United States). The Kolmogorov-Smirnov test was used to determine whether the variable had a normal distribution. Means and standard deviations (SD) were calculated for continuous and numbers (percentages) for categorical data. The differences between medical and

Table 1 Baseline Clinical Characteristics of the Surgery and Medical Group at time of diagnosis

\begin{tabular}{|c|c|c|c|}
\hline & Surgery Group $(n=29)$ & Medical Group $(n=33)$ & $p$ \\
\hline Age (years) (mean $\pm S D$ ) & $8.5 \pm 3.6$ & $8.6 \pm 2.7$ & 0.900 \\
\hline Male $n(\%)$ & $13(44.8)$ & $17(51.5)$ & 0.621 \\
\hline Duration of symptoms (years) (mean $\pm S D$ ) & $5.3 \pm 3.7$ & $4.9 \pm 2.5$ & 0.626 \\
\hline Underlying etiology $n(\%)$ & & & 0.006 \\
\hline Primary ciliary dyskinesia & $20(69.0)$ & $21(63.6)$ & \\
\hline Congenital immune deficiency & $2(6.9)$ & $3(9.1)$ & \\
\hline Postinfectious & $6(20.7)$ & 0 & \\
\hline Idiopathic & $1(3.4)$ & $9(27.3)$ & \\
\hline \multicolumn{4}{|l|}{ Anthropometric measurements } \\
\hline Weight $z$-score (mean \pm SD) & $-1.21 \pm 1.48$ & $-0.57 \pm 1.22$ & 0.133 \\
\hline Height $z$-score (mean \pm SD) & $-1.40 \pm 1.12$ & $-0.79 \pm 1.09$ & 0.033 \\
\hline BMI z-score $($ mean \pm SD) & $-0.48 \pm 1.28$ & $-0.26 \pm 1.28$ & 0.489 \\
\hline Clubbing $n(\%)$ & & & 0.048 \\
\hline Absence & $15(51.7)$ & $25(75.8)$ & \\
\hline Presence & $14(48.3)$ & $8(24.29)$ & \\
\hline Sputum culture $n(\%)$ & & & 0.502 \\
\hline No microorganism & $14(48.3)$ & $15(45.5)$ & \\
\hline H. influenzae & $12(41.4)$ & $16(48.5)$ & \\
\hline S. pneumoniae & $3(10.3)$ & $1(3.0)$ & \\
\hline M. catarrhalis & 0 & $1(3.0)$ & \\
\hline \multicolumn{4}{|l|}{ Pulmonary function tests (mean \pm SD) } \\
\hline & $(n=23)$ & $(n=31)$ & \\
\hline FEV1\% & $67 \pm 17$ & $86 \pm 16$ & $<0.001$ \\
\hline FVC\% & $77 \pm 17$ & $90 \pm 16$ & 0.007 \\
\hline FEF $25-75 \%$ & $55 \pm 24$ & $81 \pm 26$ & $<0.001$ \\
\hline
\end{tabular}

Abbreviations: FEF, forced expiratory flow; FEV, forced expiratory volume; FVC, forced vital capacity; H. influenzae, Haemophilus influenzae; M. catarrhalis, Moraxella catarrhalis; SD, standard deviation; S. pneumoniae, Streptococcus pneumoniae. 
surgical groups were compared with an independent $t$-test. Chi-squared test was performed to compare proportions between groups. The correlations between PFT and anthropometric data were analyzed by Pearson's correlation. Repeated measures of variance analyses were used for yearly anthropometric and laboratory changes of patients. Statistical tests were two-sided, and statistical significance was accepted at $p<0.05$.

\section{Results}

Medical records of 29 patients in the surgery group and 33 age- and gender-matched patients in the medical group were analyzed. When the baseline data were evaluated, it was found that mean age of patients was $8.5 \pm 3.6$ years (range $2-14.2$ years) in the surgery group and $8.6 \pm 2.7$ years (range 4.2-13.7 years) in the medical group. The mean duration of symptoms before diagnosis was 5.3 years (range 3 months to 14 years) in the surgery group and 4.9 years (range 6 months to 10 years) in the medical group. Descriptive baseline characteristics of the surgery group and medical group are displayed in - Table 1.
The most common affected sites were the left lower lobe (72.4\%), the right middle lobe $(41.4 \%)$, the right lower lobe (34.5\%), and the left lingula (34.5\%) in the surgery group and the left lower lobe (72.7\%), the right middle lobe (51.5\%), the right lower lobe (39.4\%), and the left lingula (21.2\%) in the medical group. There was no statistical significance on lobar distribution of bronchiectasis, in both groups (-Table 2 ).

The mean follow-up time of patients was 7.7 years in the surgery group and 6.8 years in the medical group after the diagnosis. In the surgery group, the mean follow-up time after the surgery was 5.5 years.

At the time of diagnosis, there was a positive correlation between weight $z$-score, height $z$-score, BMI $z$-score, and all PFT parameters, and this correlation was all statistically significant except correlation between height $z$-score and FVC\% and FEF25-75\% (- Table 3).

In the surgery group, the most common lung resections were the left inferior lobectomy (65.5\%) and the right middle lobectomy (37.9\%); two patients underwent left pneumonectomy (-Table 4). Five patients underwent second lobectomy of the other bronchiectatic lobe because of recurrent

Table 2 Localization of Bronchiectasis in the Surgery and Medical Group

\begin{tabular}{|c|c|c|c|c|c|c|}
\hline & & Surgery & group $n(\%)$ & & & $p$ \\
\hline Underlying etiology & PCD & Immune deficiency & Postinfectious & Idiopathic & Total $n(\%)$ & \\
\hline \multicolumn{7}{|l|}{ Lobe involvement } \\
\hline Right superior & $1(14.3)$ & $1(14.3)$ & $4(57.1)$ & $1(14.3)$ & $7(24.1)$ & 0.217 \\
\hline Right middle & $8(66.7)$ & $1(8.3)$ & $3(25)$ & 0 & $12(41.4)$ & 0.425 \\
\hline Right lower & $8(80)$ & 0 & $2(20)$ & 0 & $10(34.5)$ & 0.690 \\
\hline Left superior & $5(71.4)$ & 0 & $2(28.6)$ & 0 & $7(24.1)$ & 0.124 \\
\hline Left lingula & $10(100)$ & 0 & 0 & 0 & $10(34.5)$ & 0.243 \\
\hline Left lower & $16(76.2)$ & $1(4.8)$ & $3(14.3)$ & $1(4.8)$ & $21(72.4)$ & 0.978 \\
\hline One lobe involvement & $2(50)$ & $1(25)$ & $1(25)$ & 0 & $4(13.7)$ & 0.193 \\
\hline Two lobe involvement & $7(58.3)$ & $1(8.3)$ & $3(25)$ & $1(8.3)$ & $12(41.3)$ & 0.747 \\
\hline \multirow[t]{2}{*}{ Three lobe involvement } & $11(84.6)$ & 0 & $2(15.4)$ & 0 & $13(44.8)$ & 0.149 \\
\hline & & Medical & group $n(\%)$ & & & \\
\hline Underlying etiology & PCD & Immune deficiency & Postinfectious & Idiopathic & Total $n(\%)$ & \\
\hline \multicolumn{7}{|l|}{ Lobe involvement } \\
\hline Right superior & $2(50)$ & 0 & 0 & $2(50)$ & $4(12.1)$ & \\
\hline Right middle & $12(70.6)$ & $1(5.9)$ & 0 & $4(23.5)$ & $17(51.5)$ & \\
\hline Right lower & $8(61.5)$ & $1(7.7)$ & 0 & $4(30.8)$ & $13(39.4)$ & \\
\hline Left superior & $2(50)$ & 0 & 0 & $2(50)$ & $4(12.1)$ & \\
\hline Left lingula & $5(71.4)$ & $2(28.6)$ & 0 & 0 & $7(21.2)$ & \\
\hline Left lower & $15(62.5)$ & $3(12.5)$ & 0 & $6(25)$ & $24(72.7)$ & \\
\hline One lobe involvement & $5(55.6)$ & $1(11.1)$ & 0 & $3(33.3)$ & $9(27.2)$ & \\
\hline Two lobe involvement & $11(73.3)$ & $1(6.7)$ & 0 & $3(20)$ & $15(45.4)$ & \\
\hline Three lobe involvement & $5(55.6)$ & $1(11.1)$ & 0 & $3(33.3)$ & $9(27.2)$ & \\
\hline
\end{tabular}

Abbreviation: $\mathrm{PCD}$, primary ciliary dyskinesia.

Statistical significance is accepted at $p<0.05$. 
Table 3 Correlation between PFT and anthropometric data of all patients (both surgery and medical groups) at time of diagnosis

\begin{tabular}{|l|l|l|l|}
\hline PFT parameters & $\begin{array}{l}\text { Weight z-score } \\
r / p^{a}\end{array}$ & $\begin{array}{l}\text { Height z-score } \\
r / p^{a^{a}}\end{array}$ & $\begin{array}{l}\text { BMI z-score } \\
r / p^{\mathrm{a}}\end{array}$ \\
\hline FEV1 \% & $0.491 / 0.002$ & $0.293 / 0.032$ & $0.447 /<0.001$ \\
\hline FVC\% & $0.500 / 0.002$ & $0.256 / 0.061$ & $0.431 / 0.001$ \\
\hline FEF25-75\% & $0.367 / 0.026$ & $0.253 / 0.065$ & $0.283 / 0.038$ \\
\hline
\end{tabular}

Abbreviations: BMI, body mass index; FEF, forced expiratory flow; FEV, forced expiratory volume; FVC, forced vital capacity; $r$, correlation coefficient. $r / p^{\mathrm{a}}$ : correlation coefficient $/ p$ value.

pulmonary exacerbations in spite of medical treatment during their follow-up. The selected surgical approach in all cases was lateral thoracotomy.

Among 29 patients in the surgery group, 24 patients' anthropometric data were available for 2 years postoperatively. The mean age for surgery was 10.7 years (range 2-17 years). Mean values of height $z$-score were $-1.68 \pm 0.92$ at the time of

Table 4 Surgical Approach to Patients

\begin{tabular}{|l|l|}
\hline Surgical Approach & $\boldsymbol{n}(\%)$ \\
\hline Right superior lobectomy & $3(10.3)$ \\
\hline Right middle lobectomy & $11(37.9)$ \\
\hline Right lower lobectomy & $7(24.1)$ \\
\hline Left superior lobectomy & $4(13.8)$ \\
\hline Left lingula lobectomy & $7(24.1)$ \\
\hline Left lower lobectomy & $19(65.5)$ \\
\hline Unilobectomy & $11(37.9)$ \\
\hline Bilobectomy & $14(48.2)$ \\
\hline Lobectomy + Segmentectomy & $2(6.8)$ \\
\hline Left pneumonectomy & $2(6.8)$ \\
\hline
\end{tabular}

surgery and improved to $-1.42 \pm 1.22$ and $-1.34 \pm 1.05$ in the first and second postoperative years, respectively ( $p=0.013$ ); however, mean BMI $z$-score value which was $-0.91 \pm 1.14$ at the time of surgery decreased to $-1.02 \pm 1.51$ and $-1.03 \pm 1.50$ in the first and second postoperative years, respectively, which was not statistically significant (-Table 5 ).

In the surgery group, only 15 patients could perform PFT in postoperative 2 years. Mean values of FEV1\%, FVC\%, and FEF25-75\% at the time of surgery decreased in the first postoperative year, which were statistically significant, but, then, increased in the second postoperative year. The mean values of PFT parameters in the postoperative second year were less than the values at the time of surgery, but these values were statistically not significant (-Table 5).

Among 29 patients in the surgery group, only 22 patients' data about the number of pulmonary exacerbations and antibiotic requirements in 1 year were available. There was no difference on mean annual exacerbation frequency in 1 year and oral antibiotic requirements at the time of surgery and in the first and second years postoperatively; however, annual intravenous antibiotic requirements decreased significantly from $0.95 \pm 0.90$ at the time of surgery to $0.45 \pm 0.74$ and $0.18 \pm 0.66$ in the first and second postoperative years, respectively $(p=0.001)$ (-Table 5).

Table 5 Changes in Clinical Course of the Patients in the Surgery Group

\begin{tabular}{|l|l|l|l|l|l|}
\hline mean \pm SD & $n^{*}$ & At time of surgery & $\begin{array}{l}\text { Postoperative } \\
\text { first year }\end{array}$ & $\begin{array}{l}\text { Postoperative } \\
\text { second year }\end{array}$ & \begin{tabular}{l}
$p$ \\
\hline Age (years)
\end{tabular} \\
\hline Height z-score & 24 & $10.7 \pm 3.4$ & $11.8 \pm 3.4$ & $12.8 \pm 3.4$ & $<\mathbf{0 . 0 0 1}$ \\
\hline BMI z-score & 24 & $-1.68 \pm 0.92^{\mathrm{a}}$ & $-1.42 \pm 1.22^{\mathrm{b}}$ & $-1.34 \pm 1.05^{\mathrm{b}}$ & $\mathbf{0 . 0 1 3}$ \\
\hline FEV1\% & 24 & $-0.91 \pm 1.14$ & $-1.02 \pm 1.51$ & $-1.03 \pm 1.50$ & 0.814 \\
\hline FVC\% & 15 & $66.7 \pm 15.6^{\mathrm{a}}$ & $57.1 \pm 16.1^{\mathrm{b}}$ & $61.5 \pm 14.7^{\mathrm{a}, \mathrm{b}}$ & $\mathbf{0 . 0 1 2}$ \\
\hline FEF25-75\% & 15 & $74.7 \pm 13.9^{\mathrm{a}}$ & $65.9 \pm 17.8^{\mathrm{b}}$ & $69.3 \pm 18.2^{\mathrm{a}, \mathrm{b}}$ & $\mathbf{0 . 0 2 3}$ \\
\hline Annual exacerbation frequency & 15 & $52.1 \pm 18.0^{\mathrm{a}}$ & $40.5 \pm 13.6^{\mathrm{b}}$ & $43.8 \pm 13.7^{\mathrm{b}}$ & $\mathbf{0 . 0 0 6}$ \\
\hline Annual intravenous antibiotic requirement & 22 & $1.91 \pm 0.38$ & $1.09 \pm 0.34$ & $1.14 \pm 0.70$ & 0.050 \\
\hline Annual oral antibiotic requirement & 22 & $0.91 \pm 1.19$ & $0.64 \pm 1.14$ & $0.91 \pm 1.51$ & 0.617 \\
\hline
\end{tabular}

Abbreviations: BMI, body mass index; FEF, forced expiratory flow; FEV, forced expiratory volume; FVC, forced vital capacity; SD, standard deviation. ${ }^{a}$ Means within a row followed by the same superscript are not significantly different.

${ }^{\mathrm{b}}$ Means within a row followed by the same superscript are not significantly different.

*Number of patients whose data are available. 


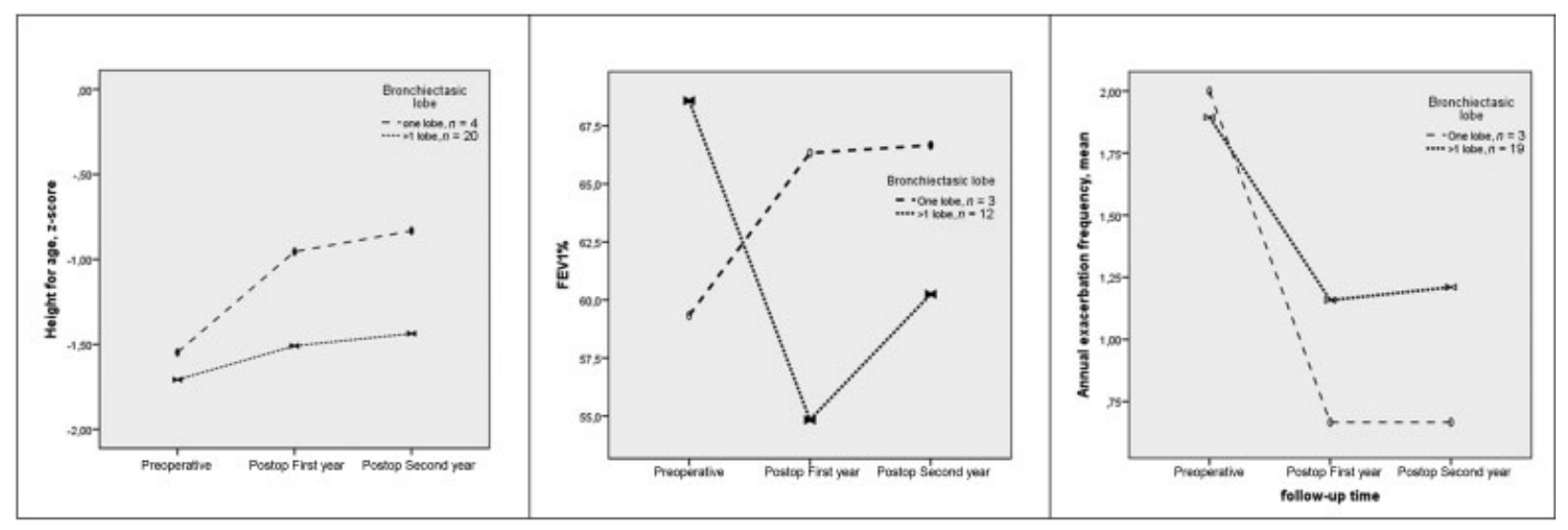

Fig. 1 Height $z$ scores, FEV1\% values, and annual exacerbation frequencies in patients who had one lobe affected and in patients who had more than one lobe affected in the surgically treated group.

We also evaluated the outcomes of patients who had one lobe affected with those of patients who had more than one lobe affected in the surgically treated group. Height $z$-scores in the baseline and postoperative first and second years were available in four patients with one lobe affected and in 20 patients with more than one lobes affected. FEV1\% values and annual exacerbation frequencies in these periods were available in 3 patients with one lobe affected and in 12 patients with more than one lobes affected and in 3 patients with one lobe affected and in 19 patients with more than one lobes affected, respectively. Therefore, we were not able to make statistics due to small number of data; however, the comparison of these two groups showed that height for $z$-scores, FEV1\% values, and annual exacerbation frequencies improved better in patients with one lobe affected at the end of the postoperative second year than in patients with more than one lobe affected (- Fig. 1).

Among 33 patients in the medical group, 30 patients' anthropometric data were available for 3 years. In this group, mean BMI $z$-scores decreased gradually and statistically significantly from the baseline toward the third year, which was $-0.33 \pm 1.31$ in the beginning and $-0.63 \pm 1.51$ at the end $(p=0.033)$. Height $z$-score mean value statistically insignificantly increased from the baseline which was $-0.84 \pm 1.08$ to $-0.83 \pm 1.20,-0.66 \pm 1.18$, and $-0.72 \pm 1.41$ in the first, second, and third years of medical treatment, respectively (-Table 6).

In the medical group, 15 patients performed PFTs; mean FEV1\%, FVC\%, and FEF25-75\% did not change significantly within years (-Table 6 ).

Among 33 patients in the medical group, data about the number of pulmonary exacerbations and antibiotic requirements in 1 year were available only in 24 patients; mean annual exacerbation frequency, annual intravenous, and oral antibiotic requirements decreased significantly in 3 years from $2.38 \pm 1.37$ to $0.96 \pm 0.91$, from $1.12 \pm 1.19$ to $0.13 \pm 0.34$, and from $1.25 \pm 0.94$ to $0.83 \pm 0.95$, respectively ( - Table 6 ).

Table 6 Changes in Clinical Course of the Patients in Medical Group

\begin{tabular}{|l|l|l|l|l|l|l|}
\hline mean \pm SD & $\boldsymbol{n}$ & Baseline & First year & Second year & Third year & $\boldsymbol{p}$ \\
\hline Age (years) & 30 & $8.2 \pm 2.5$ & $9.3 \pm 2.5$ & $10.3 \pm 2.5$ & $11.4 \pm 2.4$ & $<\mathbf{0 . 0 0 1}$ \\
\hline Height z-score & 30 & $-0.84 \pm 1.08$ & $-0.83 \pm 1.20$ & $-0.66 \pm 1.18$ & $-0.72 \pm 1.41$ & 0.132 \\
\hline BMI z-score & 30 & $-0.33 \pm 1.31^{\mathrm{a}, \mathrm{b}}$ & $-0.31 \pm 1.27^{\mathrm{a}}$ & $-0.52 \pm 1.31^{\mathrm{b}}$ & $-0.63 \pm 1.51^{\mathrm{b}}$ & $\mathbf{0 . 0 3 3}$ \\
\hline FEV1\% & 15 & $87.2 \pm 15.4$ & $82.9 \pm 13.4$ & $83.1 \pm 10.1$ & $81.9 \pm 14.7$ & 0.367 \\
\hline FVC\% & 15 & $90.5 \pm 15.0$ & $89.5 \pm 13.7$ & $90.0 \pm 10.2$ & $88.3 \pm 14.6$ & 0.903 \\
\hline \multicolumn{1}{|c|}{ FEF25-75\% } & 15 & $81.5 \pm 28.5$ & $80.2 \pm 26.1$ & $77.0 \pm 24.0$ & $78.9 \pm 34.7$ & 0.895 \\
\hline Annual exacerbation frequency & 24 & $2.38 \pm 1.37^{\mathrm{a}}$ & $1.13 \pm 0.95^{\mathrm{b}}$ & $0.88 \pm 0.90^{\mathrm{b}}$ & $0.96 \pm 0.91^{\mathrm{b}}$ & $<\mathbf{0 . 0 0 1}$ \\
\hline $\begin{array}{l}\text { Annual intravenous } \\
\text { antibiotic requirement }\end{array}$ & 24 & $1.12 \pm 1.19^{\mathrm{a}}$ & $0.37 \pm 0.65^{\mathrm{b}}$ & $0.13 \pm 0.45^{\mathrm{b}}$ & $0.13 \pm 0.34^{\mathrm{b}}$ & $<\mathbf{0 . 0 0 1}$ \\
\hline Annual oral antibiotic requirement & 24 & $1.25 \pm 0.94^{\mathrm{a}}$ & $0.75 \pm 0.79^{\mathrm{b}}$ & $0.75 \pm 0.79^{\mathrm{b}}$ & $0.83 \pm 0.95^{\mathrm{a}, \mathrm{b}}$ & $\mathbf{0 . 0 4 2}$ \\
\hline
\end{tabular}

Abbreviations: BMI, body mass index; FEF, forced expiratory flow; FEV, forced expiratory volume; FVC, forced vital capacity; SD, standard deviation. ${ }^{a}$ Means within a row followed by the same superscript are not significantly different.

${ }^{b}$ Means within a row followed by the same superscript are not significantly different.

Notes: $n$ indicates the number of patients whose data are available. Bold means $p<0.05$ and it is statistically significant. 


\section{Discussion}

Our study is the first study, which evaluates longitudinal growth together with pulmonary functions and annual exacerbation rates of surgically treated children with nonCF bronchiectasis and compares it with only medically treated patients. We found that over 2 years, height $z$-score improved, and annual intravenous antibiotic requirements decreased in surgically treated children; BMI $z$-scores, annual exacerbation frequencies, oral antibiotic requirements, and pulmonary functions did not change significantly. However, in the medically treated group, both annual exacerbation frequencies and oral and intravenous antibiotic requirements decreased; height $z$-scores showed non-significant improvement, BMI $z$-scores decreased over 3 years, and pulmonary functions remained almost stable.

In our study, age- and gender-matched 29 surgically and 33 medically treated children were included; at the time of diagnosis, the durations of symptoms were similar to each other, but we noticed that in physical examination, clubbing was significantly much more present in surgically treated patients. Besides, nutritional status defined by weight $z$-scores, height $z$-scores, and BMI $z$-scores were all in the normal range (between -2 and +2 ) in both groups, but all of them were lower in the surgery group, which was statistically significant in height $z$-scores only. Baseline spirometric values were also significantly lower in the surgical group at the time of diagnosis. Therefore, these findings retrospectively showed that the patients whom we decided to operate had poorer nutritional state and had "severe disease" characterized by prominent clubbing and abnormal pulmonary functions at referral.

Indications of surgery are not established fully in children yet. However, it is recommended that children with severe, poorly controlled, localized disease or recurrent hemoptysis may require surgical resection of a bronchiectatic lobe. ${ }^{1,2,12}$ In our study, most patients in the surgery group had more than one lobe involvement, and there were also patients with localized disease who were only medically treated just because of subjective measures like growth of the patient, pulmonary functions, and clinical findings, including annual exacerbations in their follow-up. Therefore, decision to operate children with localized bronchiectasis needs to be clarified.

Patients with a lower BMI were found to be prone to developing more acute exacerbations and worse pulmonary function, which is a major determinant of hospitalization and death risks in non-CF bronchiectasis. ${ }^{13,14}$ We also demonstrated that there was a positive correlation between baseline weight $z$-score, height $z$-score, and BMI $z$-score and all PFT parameters in both groups, and this correlation was all statistically significant with FEV1\%, which means better growth. Nutritional status is strongly associated with better pulmonary functions, and if nutritional status is getting worse, pulmonary functions are declining. Nutritional status of patients with CF is known to be strongly associated with pulmonary function, respiratory status, and survival. ${ }^{15,16}$ Similar correlation in our findings with non-CF bronchiec- tasis emphasizes that maximum efforts should be performed to improve nutritional status of patients with non-CF bronchiectasis, as well.

In the literature, there are numerous reports about the outcome of surgical intervention of both adult and pediatric patients with non-CF bronchiectasis, and they were systematically reviewed in a meta-analysis recently. ${ }^{17}$ In this metaanalysis, it was concluded that lung resection in the management of non-CF bronchiectasis was associated with significant improvements in symptoms, low risk of mortality, and acceptable morbidity. ${ }^{17}$ In a study from our country in which children undergoing resection for bronchiectasis were evaluated, $64.7 \%$ were asymptomatic, and $23.5 \%$ had improved compared with their preoperative symptoms in the followup period which was at least 3 years after the surgery. However, clinical improvement was defined as a decrease in symptoms of infection episodes when compared with the number of episodes without any objective numbers. ${ }^{18}$ Again, from another study in children with bronchiectasis who were surgically managed, postoperative outcomes were assessed by asking the patients and were categorized as "perfect" if they had no symptoms postoperatively; "improved" if they had symptoms, but milder than preoperative period; and "no change," and they found that the outcome was "perfect" in $73.3 \%$, "improved" in $23.3 \%$, and "no changes" in $3.4 \%$ cases in a mean follow-up of 4.3 years after surgery; however, their outcome parameters were totally subjective. ${ }^{19}$ In another study, again from Turkey, the annual lower respiratory infection rate decreased, and lung function tests were improved in both surgically treated and medically treated patients; however, outcome was reported as annual rate of exacerbations, and PFT and parent/patient report of improvement were the same in both groups during follow-up period of 4.7 years on average. $^{20}$ In none of those studies, longitudinal growth of surgically treated children with non-CF bronchiectasis was reported, and our report is the first study in these patients.

There is little information about longitudinal growth of children with non-CF bronchiectasis. In a study by Bastardo et $\mathrm{al}^{21}$ it was found that, in children with non-CF bronchiectasis with different etiologies, there was improvement in height $z$-scores, but no improvement in weight $z$-scores and BMI $z$-scores with standard medical treatment in 4 years, and they showed adequate growth in time. In another study by Kapur et $\mathrm{al}^{22}$ in which lung function and growth in children with non-CF bronchiectasis were longitudinally reviewed, BMI $z$-score improved, but height $z$-score showed non-significant improvement with medical treatment, and it was concluded that anthropometric parameters in these children with non-CF bronchiectasis remain stable over a 3- to 5-year follow-up period once appropriate therapy is instituted. In our study, although statistically insignificant, height $z$-score of patients in the medical group improved as in the abovementioned studies with standard medical treatment; however, the improvement in the height $z$-scores in the surgery group was statistically significant, which was not explained in the literature before; this much prominent increase can be attributed to the statistically significant lower baseline 
values at the time of diagnosis in the surgical group than in the medical group. They benefitted from surgery in means of height although their final height $z$-scores at the end of the study were still lower than those in the medical group in spite of standard medical treatment in the postoperative period, as well. We could not evaluate longitudinal weight $z$-scores of our patients because weight $z$-scores were not available for patients older than 10 years in the WHO AnthroPlus packet program; therefore, we could evaluate only BMI $z$-scores. BMI $z$-scores of our patients did not improve in the surgery group; this may mean that these patients did not gain weight parallel to improvement of their height. Furthermore, BMI $z$-scores of our patients in the medical group decreased significantly, which reflects inadequate weight gain in spite of a decrease in their annual exacerbation rate and antibiotic use.

It has been demonstrated that pulmonary function of children with non-CF bronchiectasis declines significantly over a 4.5 year period, despite treatment in a similar pattern to $\mathrm{CF}^{23}$ In the study by Bastardo et al, ${ }^{21}$ children with non-CF bronchiectasis were found to have stable lung function $z$-scores with standard medical treatment in 4 years, and Kapur et $\mathrm{al}^{22}$ demonstrated stable spirometric parameters over a 3- to 5-year follow-up period once appropriate therapy is instituted in children with non-CF bronchiectasis, and severe exacerbations resulted in accelerated lung function decline. Similarly, we showed stable pulmonary functions in follow-up years of both groups. In medical group, the initiation of standard medical treatment led to significant decrease in annual exacerbation rate and antibiotic use, which caused stabilization and at least prevented deterioration of their pulmonary functions. Also in the surgery group, after the operation, with the significant decrease in hospitalization and intravenous antibiotic use, the pulmonary function of patients were shown to be stabilized in the second postoperative year in spite of a decrease in the first postoperative year in the surgery group, which may be the reason for stabilization of their lung functions. In some studies, comparison between pre- and postoperative lung function test values showed that surgery influenced neither FVC nor FEV1, despite parenchymal resection. ${ }^{24-26}$

The main duration of symptoms was 5.3 years in the surgery group. At the baseline, patients who had surgery had worse anthropometric parameters, worse PFT, and more clubbing than had medically treated patients. Therefore, although we do not have enough data, we believe that patients would have had much more benefit if they had surgery earlier, before their growth and pulmonary functions declined. Therefore, close monitoring of the clinical and lung function parameters and deciding surgery in the earlier period is important in children with non-CF-bronchiectasis, as earlier surgery in appropriate patients could lead to even better results.

In our study, we evaluated annual exacerbation rate as well as intravenous antibiotic use, which reflect severe exacerbations that require hospitalization of patients as outcome parameters. Surgically treated patients did not show a significant decrease in the annual number of exacer- bation rate, but surgery caused a significant decrease in the hospitalization rate, which is a very important parameter of life quality of a growing child. We do not know whether those children would have a decline in their pulmonary functions and cannot predict their longitudinal growth if they had not been operated because our study also included patients with localized disease in the medical group whose pulmonary functions were preserved with only medical treatment. Surgical treatment did not cure the disease in all of our patients, may be because some had incomplete resection due to multilobar involvement in our study group, which consisted of PCD most commonly, which is a chronic disease, and the inflammation is still going on after the operation as well. Resection of the most involved part of the lung parenchyma alone may reduce the severity of post-resection symptoms, but low-grade symptoms may persist. ${ }^{27}$ The decision for operation is easier in children with a localized bronchiectasis with an underlying etiology, such as foreign body aspiration, but it is harder to decide whether to operate the patients with localized disease, such as PCD or congenital immune deficiencies when there is "severe and poorly controlled disease" in spite of courses of regular medical treatment. In our study, we retrospectively found that we decided to operate patients who had worse pulmonary functions and lower anthropometric data at diagnosis; patients with better basal pulmonary functions and better anthropometric data at diagnosis were decided to be treated only medically even though they had localized disease. We did not include the annual exacerbation rate before the operation and did not compare it to the postoperative period due to lack of our data.

The decision for surgery in patients with more than one bronchiectatic lobe is challenging. Such children can have poor prognosis, so many pulmonary exacerbations, and failure to thrive in spite of medical therapy and, therefore, may need resection of the most affected lobe to reduce the risk of infections and to catch normal growth. Although our study is retrospective, and the data are small, we demonstrated that height $z$-scores, FEV1\%, and annual exacerbation frequencies in patients were better at the end of the postoperative second year in patients with one lobe affected than those in patients with more than one lobe affected in the surgically treated group. However, patients with more than one lobe affected also gained benefit from surgery; they had better height $z$-scores and less annual pulmonary exacerbations at the end of the second postoperative year when compared with their baseline values. Further data are needed for the optimum timing to decide surgery in patients with more than one bronchiectatic lobe.

Our limitations were that this study was retrospective, and the two groups were different in their baseline height $z$-score and pulmonary function tests. In addition, few patients had PFT. Furthermore, lung volumes of our patients with plethysmography were not available. The other limitations were that we did not include the microbiological status of our patients whether they were chronically colonized with a microorganism or not, and we did not include radiologic scoring of HRCT of chest. Also, the study would have been much better if we had included an objective questionnaire 
about the life quality of patients in both groups, as well. Socioeconomical and environmental factors, such as housing and tobacco smoke exposure, were also not evaluated in our study, which have a very important role in the growth of children, which is a multifactorial issue. Our main aim was to evaluate the growth, clinical findings, and pulmonary functions of our surgically treated patients in the long-term; therefore, we did not intend to evaluate perioperative complications of surgery for bronchiectasis in our patients.

We conclude that longitudinal growth can be achieved, and pulmonary functions can be preserved with aggressive medical treatment starting from the diagnosis in children with non-CF bronchiectasis. Early diagnosis of bronchiectasis, determination of the underlying etiology, and institution of appropriate therapy as soon as possible are the most important factors for preventing progression of the disease. There is no doubt that patients with localized disease not responding to medical treatment are candidates of surgical treatment; however, every patient needs to be individually evaluated for this decision. The decision for lung resection in children with localized disease and especially with more than one lobe involvement should be carefully taken, and the underlying etiology should be taken into consideration. Further, larger prospective longitudinal clinical trials should be conducted to establish criteria for the indications and timing of surgery in children with non-CF bronchiectasis.

\section{Funding Source}

There is no financial source.

\section{Conflict of Interest}

There is no conflict of interest declared.

\section{Acknowledgment}

We thank Associate Professor Dr. Erdem Karabulut from Hacettepe University Faculty of Medicine Department of Biostatistics for assistance with statistical analysis.

\section{References}

1 Goyal V, Grimwood K, Marchant J, Masters IB, Chang AB. Pediatric bronchiectasis: No longer an orphan disease. Pediatr Pulmonol 2016;51(05):450-469

2 Boren EJ, Teuber SS, Gershwin ME. A review of non-cystic fibrosis pediatric bronchiectasis. Clin Rev Allergy Immunol 2008;34(02): 260-273

3 Eastham KM, Fall AJ, Mitchell L, Spencer DA. The need to redefine non-cystic fibrosis bronchiectasis in childhood. Thorax 2004;59 (04):324-327

4 Callahan CW, Redding GJ. Bronchiectasis in children: orphan disease or persistent problem? Pediatr Pulmonol 2002;33(06): 492-496

5 Stafler P, Carr SB. Non-cystic fibrosis bronchiectasis: its diagnosis and management. Arch Dis Child Educ Pract Ed 2010;95(03): 73-82

6 Brower KS, Del Vecchio MT, Aronoff SC. The etiologies of non-CF bronchiectasis in childhood: a systematic review of 989 subjects. BMC Pediatr 2014;14:4
7 Martínez-García MA, Soler-Cataluña JJ, Perpiñá-Tordera M, Román-Sánchez P, Soriano J. Factors associated with lung function decline in adult patients with stable non-cystic fibrosis bronchiectasis. Chest 2007;132(05):1565-1572

8 Chang AB, Bell SC, Torzillo PJ, et al; extended voting group. Chronic suppurative lung disease and bronchiectasis in children and adults in Australia and New Zealand Thoracic Society of Australia and New Zealand guidelines. Med J Aust 2015;202(01):21-23

9 Pasteur MC, Bilton D, Hill AT; British Thoracic Society Bronchiectasis non-CF Guideline Group. British Thoracic Society guideline for non-CF bronchiectasis. Thorax 2010;65(Suppl 1):i1-i58

10 WHO AnthroPlus for personal computers manual: software for assessing growth of the world's children and adolescents. Geneva: WHO; 2009

11 American Thoracic Society. Standardization of Spirometry, 1994 Update. Am J Respir Crit Care Med 1995;152(03):1107-1136

12 McShane PJ, Naureckas ET, Tino G, Strek ME. Non-cystic fibrosis bronchiectasis. Am J Respir Crit Care Med 2013;188(06):647-656

13 Qi Q, Li T, Li JC, Li Y. Association of body mass index with disease severity and prognosis in patients with non-cystic fibrosis bronchiectasis. Braz J Med Biol Res 2015;48(08):715-724

14 Onen ZP, Gulbay BE, Sen E, et al. Analysis of the factors related to mortality in patients with bronchiectasis. Respir Med 2007;101 (07):1390-1397

15 Stark LJ, Opipari-Arrigan L, Quittner AL, Bean J, Powers SW. The effects of an intensive behavior and nutrition intervention compared to standard of care on weight outcomes in CF. Pediatr Pulmonol 2011;46(01):31-35

16 Ramírez I, Filbrun A, Hasan A, Kidwell KM, Nasr SZ. Improving nutritional status in a pediatric cystic fibrosis center. Pediatr Pulmonol 2015;50(06):544-551

17 Fan LC, Liang S, Lu HW, Fei K, Xu JF. Efficiency and safety of surgical intervention to patients with Non-Cystic Fibrosis bronchiectasis: a meta-analysis. Sci Rep 2015;5:17382

18 Haciibrahimoglu G, Fazlioglu M, Olcmen A, Gurses A, Bedirhan MA. Surgical management of childhood bronchiectasis due to infectious disease. J Thorac Cardiovasc Surg 2004;127(05): 1361-1365

19 Sirmali M, Karasu S, Türüt H, et al. Surgical management of bronchiectasis in childhood. Eur J Cardiothorac Surg 2007;31 (01):120-123

20 Karadag B, Karakoc F, Ersu R, Kut A, Bakac S, Dagli E. Non-cysticfibrosis bronchiectasis in children: a persisting problem in developing countries. Respiration 2005;72(03):233-238

21 Bastardo CM, Sonnappa S, Stanojevic S, et al. Non-cystic fibrosis bronchiectasis in childhood: longitudinal growth and lung function. Thorax 2009;64(03):246-251

22 Kapur N, Masters IB, Chang AB. Longitudinal growth and lung function in pediatric non-cystic fibrosis bronchiectasis: what influences lung function stability? Chest 2010;138(01):158-164

23 Twiss J, Stewart AW, Byrnes CA. Longitudinal pulmonary function of childhood bronchiectasis and comparison with cystic fibrosis. Thorax 2006;61(05):414-418

24 Coutinho D, Fernandes P, Guerra M, Miranda J, Vouga L. Surgical treatment of bronchiectasis: A review of 20 years of experience. Rev Port Pneumol (2006) 2016;22(02):82-85

25 Guerra MS, Miranda JA, Leal F, Vouga L. Surgical treatment of bronchiectasis. Rev Port Pneumol 2007;13(05):691-701

26 Agasthian T, Deschamps C, Trastek VF, Allen MS, Pairolero PC. Surgical management of bronchiectasis. Ann Thorac Surg 1996; 62(04):976-978, discussion 979-980

27 Stephen T, Thankachen R, Madhu AP, Neelakantan N, Shukla V, Korula RJ. Surgical results in bronchiectasis: analysis of 149 patients. Asian Cardiovasc Thorac Ann 2007;15(04):290-296 